\title{
Article
}

\section{Apriti Cielo: the public's astronomical imagery as a key to evaluate a museum project}

\author{
Stefano Giovanardi
}

An effective communication of astronomy cannot take place without considering the view the general public has on the universe. Through a number of narrative interviews with non-experts, a research was carried out on personal cosmologies, to outline the public's heterogeneous astronomical imagery. The result is a bundle of conceptions, perceptions and attitudes which are useful to interpret the difficulties the public experiences when facing the contents of astrophysics, and to establish an ongoing dialogue.

\section{Introduction}

A necessary condition for an effective scientific communication is to establish a relation with the general public. In the case of astronomy, the great interest roused by its themes is joined by the rapidity with which research drifts away from the popular culture on the sky - a trend which could be extended to contemporary science as a whole. An issue arises from this statement: how could an effective communication on new results be implemented? Dropping information, albeit a correct one, on the audience is not enough: without a relation with the public, communication will be levelled on indoctrination - that restricts itself to transmitting a priori coded contents -, or on a sterile tone of institutional propaganda.

When discussing this issue, one should not forget that behind the collective term "public", there is a heterogeneous body of people carrying their own emotions. ${ }^{1,2,3}$ These are individuals reacting to astronomy by activating their special imagery on the universe, shaped by a personal elaboration of different influences: information by the media, cinema, art, religion, together with scientific prejudices, stereotypes, beliefs, true and false knowledge. Many of these influences have nothing to do with science. But it is drawing on this imagery that everybody shapes their own conception of the cosmos and expectations are created, which are a benchmark for any new message on the universe.,

This complex cultural, emotional, fictional heritage makes up the "initial conditions" of the public, when receiving any new stimulus from astronomical sciences.

A research was carried out in order to draw up a detailed study on the spontaneous relation between the public and astronomy and its related contents. This type of knowledge may be useful to adjust the tone and the language of various initiatives of science communication in astronomy - whatever their purpose (teaching, information, evocation, entertainment and the likes) - especially to raise our awareness on the condition of the interlocutors they are addressed to and to be able to relate to them in a constructive and dialectical way.

\section{A perception study}

This chance was given by the working group for the planning and the implementation of "Apriti Cielo", the new planetarium and astronomy-space museum of Turin. It is a centre for the popularisation of astronomy, designed on the model of a science centre, located in Pino Torinese, in the vicinity of the astronomical observatory of Turin of the National Institute of Astrophysics (Inaf). This facility, to be inaugurated in 2007, includes a museum area of about $750 \mathrm{~m}^{2}{ }^{6}$ In order to effectively design the exhibition, attention was paid also to the public's naive beliefs on the cosmos and the likes. The aim was to discover whether there are themes these images on the universe enter into conflict with, and whether 
particular cognitive obstacles emerge within the themes dealt with in the exhibitions: in other words, the aim was to exploit the scientific imagery as a key-instrument for a preliminary evaluation on the exhibition project. In the museum practice jargon, this is a front-end evaluation.

The research involved twenty people, aged 15-81, chosen to have different social, professional and cultural backgrounds, who were asked to describe their view of the universe. The only relevant criterion in choosing the people was to select exclusively those who admitted they "were illiterate about it"; in short, those stating they had neither a specific interest for astronomy, nor a knowledge about this subject: our interest focussed on naive opinions, therefore experts were excluded from this research. As the Apriti Cielo project is addressed mainly to a public of high school students, the choice of the participants has fallen mostly within the 15-18 age group, representing approximately $2 / 3$ of the entire group. The following analysis has therefore focussed on a public comprising students and young adults.

The aim of this survey was not a quantitative definition of the astronomical perception, but a qualitative analysis of some of its most relevant features that may only emerge from an in-depth discussion.

\section{The interviews}

In order to establish a positive relation with the participants and to maintain an attitude which could allow them to activate their imagery, it was decided that narrative interviews should be carried out, presenting the interviewees with an outline of themes to be used as an inspiration for discussion. The dialectical set-up of the interview is the strategy adopted to keep issues "alive", that is to actively involve the interlocutor and to put them in a position to develop those conceptions that, according to cognitive psychology, ${ }^{7}$ are indicators of an underlying scenario they draw on to formulate an answer. This approach falls within the tradition of the studies on the visitors and the evaluation of the effectiveness of exhibitions. $^{8}$

In carrying out the interviews, any lexical contamination was avoided: words, nomenclatures or concepts unrelated to the ones already expressed by the interviewees were deliberately left out. In other words, not to modify the intrinsic expressivity of their language, terms differing from those already used by the interviewees have been avoided. Conforming to the interlocutor's vocabulary is the key to understand the genuine expression of their imagery.

The themes of the interviews were the following: What is your personal relation with the sky? What do you think astronomy deals with? Can you tell me what an astronomer does? What is your view of the universe? Is it finite or infinite? Do you think universe changes over time? How is universe observed? Would you travel into space? Where would you go?

The questions were chosen according to the issues that will be dealt with by the Apriti Cielo exhibition (What is the Universe like? How does the Universe work? How is it evolving? How is its observation carried out?).

Starting from this blueprint and supporting the attitude spontaneously adopted by the participants, each interview was carried out for about an hour, highlighting any detail as much as possible. All of the surveyed people took seriously their participation in the interviews. This crucial element helped to focus the discussion on the consequences of the statements expressed and, in many cases, to trace their origins to the imagery. In particular, this in-depth analysis was useful in the process to identify the mechanisms underlying the imagery activation, which are frequently recognisable in the impromptu formulation of ad hoc conceptions, ready to be transformed or abandoned when facing subsequent difficulties, but useful to overcome any crisis points created by a question.

An element emerging on a large scale is the embarrassment generated by the request to discuss in a non-superficial way the major issues of astronomy. The creativity of some answers is a symptom of a lack of familiarity with the subject - which was predictable, given the characteristics of the interviewee sample - but also of a struggling search for references to frame this issue. What emerges is the need for an authoritative figure able to suggest possible cultural links with astronomy, a sensible mediator willing to take on the role of a scientific critic, whose action may, in the end, make information meaningful and become part of a shared culture. 


\section{Analysis of personal cosmologies}

These interviews reveal a considerable variety of views, analysed as doors to the imagery. Quite significantly, some conceptions are recurring, regardless of the people's background. Some of the most interesting ones will be illustrated below in order to sketch an outline - or most probably to draw a picture - of the general public's imagery on astronomy. The quotations which follow are literally translated from Italian.

\section{Personal relation with the sky}

When asked to turn their eyes to the sky, people fall into two categories, divided by the psychological attitude towards the universe: enthusiasts and "chicken". In general, nearly nobody is exempt from the charm of the firmament (albeit some are uninterested and some essentially have a meteorological relation with the sky), and there are two evenly-proportioned groups: those who would be enthusiastic about a journey in the cosmos - either excited by the pleasure of a discovery or by the chance to find better worlds in the sky - and those who feel scared and anguished before the infinite: "After a while I feel distressed by the idea of the infinite." (Giulia, 34, actress).

\section{Astronomy and the astronomer}

Astronomy is considered as a human and imperfect science. The effort by the astronomer is to forget the natural laws of planet Earth and to imagine extreme situations that may occur in outer space only. This concept is very interesting because, although founded on a wrong principle (the non-generality of physical laws), it contains an element of truth, that is the existence of an astrophysical environment with extreme characteristics, which places "laboratories" - otherwise not available on Earth - at the disposal of astronomers. It will suffice to mention the recent discovery of a dual-line pulsar and the chance to exploit it to verify the theory of relativity and the gravitational-wave emission. ${ }^{9}$

The figure of the astronomer inspires admiration among the public. Even though sometimes he or she is described as a scientist busy working on his or her computer rather than on complicated mathematical maps, the image of the astronomer is still strongly linked to the classic Galilean stereotype, envisaging a scientist patiently leaning onto his telescope to scan the dark sky. Indeed, the main qualities connected to an astronomer are his/her ability to observe, patience and accuracy in identifying the details. But also his/her abstraction ability and passion for his/her work. Quite surprisingly, the astrophysicist is apparently judged as a "less scientific" and a more "down-to-earth" figure than the astronomer, and this is probably due to a lack of references on this new profession.

\section{The Universe: finite or infinite?}

A great debate is provoked by the issue of the finite or infinite universe: although everybody agrees on the concept of infinite, the efforts imagination makes to represent it are complex and sometimes require some logical strides. "The universe is infinite. [...] And the stars are at three fourths of it" (Antonio, 18, scientific secondary school student). These actually are interesting passages for our research, as they mark precisely the moments activating the production (or the recollection) of a conception believed to be appropriate for the issue proposed: "Finite or infinite. Hum. I think it is infinite. And I am referring especially to my idea of black holes" (Valentina, 18, scientific secondary school student).

In fact, imagining the borders of a universe believed to be infinite, but frequently compared with approximation to an immense sphere is not a trivial problem for imagination, and it leads to different and expressive solutions: "Sometimes I wonder, looking at the sky, how its end may be, because it is unknown. Then you imagine that, from completely dark, it suddenly turns into completely white and there is nothing there..." (Isabella, 15, classical secondary school student). 


\section{A gentle giant}

Either vast or immense, the universe is conceived by some also as infinitely gentle: almost comparable to a living thing whose changes are due to its free will. "The universe... how do I imagine it? As a gentle giant" (Luisa, 15, scientific secondary school student). The effects of physics (motions, evolution) are interpreted as signs of a living will (implying an ability to choose). This creates a biological short circuit with potentially profound consequences. It spreads on the determinism of gravitational motion, on the life cycles of stars, up to explain even the universe evolution as a conscious answer to a space selforganisation need, e.g. to make room for its components: "The stars, there may be 2,000 stars, but maybe more. However, if you put 100 of them here, 200 there and further 300 there, maybe then they could not fit into this sphere. And therefore this sphere may need to enlarge to make them fit more... comfortably" (Antonio, 19, scientific secondary school student).

\section{Vague stars, vague ideas}

Even the stars, albeit more definite objects than the universe as a whole, exert contrasting influences on the public. This results in very peculiar perceptions, such as the idea that stars may be cold: "Maybe it could be a warm light because it burns for its warmth, or it may be a light burning because of too much ice. Maybe it is burning precisely because a star shines a light, although with reverse reactions, rather ice-cold than hot." (Valentina, 18, scientific secondary school student).

Cold or warm, stars are suspended in space, or - even better - they "float". The need to provide the stars with a support reveals a conjecture which is implicitly formed, that is placing stars in a reference system with a downward-oriented gravity. It is the only one - according to many physics teachers at secondary schools - that students are able understand: planet Earth, with its ever downward-oriented gravity.

\section{The winning system}

All of these interviews share a common feature, whose frequency leads us to draw conclusions broader than our sample, as confirmed by the comparison with other studies on the visitors to astronomical museums..$^{10}$ The representation of the universe elaborated by the public through the instruments provided by a popular imagery is still essentially centred on a comfortable cosmos made of stars and planets, with a large dark area around it, which is the door to the borders of the universe. In other words, the awareness on the space in which we live is limited to our solar neighbourhood.

In fact, very frequently in popular cosmology, the prevalence of the Solar System as a reference framework for the organisation of celestial hierarchies is able to overcome and include also concepts such as stars, galaxies and, for some, even the entire universe. A well-founded suspicion is that this happens just because the Solar System, is precisely a "system". This is a strong term, able to evoke immediately a paradigm, an order, a recognisable and trustful regularity. This may be a sufficient guarantee to many, who easily associate planet orbits to the trajectories of cosmic motions and see this eternal and all-encompassing circularity as the "quadrature" of the sky. Similarly, and partially as a consequence, the role played by the Sun is perceived as the prevailing one among all of the celestial bodies, really out of proportion to its actual role: even though nobody places it at the centre of the universe, many see it as the body lighting all the stars and the planets. Without the Sun, the universe dies: the end of the universe or of our galaxy is frequently believed to be happening with the death of the Sun.

An imagination void starting just outside the Solar System is thus unveiled: a lack of references able to drive popular perception through the sidereal spaces, up to encountering galaxies and the universe on a broader scale.

Further beyond, there is an endless limbo reaching the unimaginable borders of the universe. A probable consequence of this void is that the majority of the interviewees have no idea neither of the actual dimensions under discussion (six months is the estimated time needed to reach a star), nor of the real challenges of contemporary astrophysics (the majority mention generic studies on the planets, meteorites, stars). Without the slightest hint of a popular perception of the universe on a large scale, but even of galaxies, it must be very hard for the general public to grasp the actual dimensions of the current 
research and of the great issues opened up by cosmology. As hard as acknowledging the need for more and more sophisticated instruments, generically associated with the inevitability of the technological progress. Indeed, questioned on the reasons behind the need for telescopes and instruments to observe the space, the majority of the public state that it is because "the stars can be seen more from close up".

\section{Conclusions}

Taking the public's general perception on astronomy into account is a sign of maturity in science communication, especially in a time when research stretches up to horizons which are very distant from the sky the people are familiar with.

As far as the setting-up of Apriti Cielo is concerned, the main result of this evaluation was a very radical change in the choice of the subjects to be dealt with: the planets and the Solar System were removed from the four floors of the exhibition, whereas the original project envisaged large models, dioramas to be hung to the ceiling and interactive installations. ${ }^{11}$ The whole exhibition area was devoted to cosmology and modern astrophysics instead.

Indeed, we thought that the most important indication to be extracted from this research is that speaking about galaxies and cosmology will be the greatest challenge of science information dissemination, because it will have to address a public that is absolutely puzzled beyond the reassuring circles of the Solar System. Meeting this challenge is worthwhile, as it may be one of the most interesting achievements to open up a new phase in the communication of astronomy, to finally teach the public how to look further. Out there, without the comforting warmth of the Sun, the traditional astronomy power ends and there comes the one by modern astrophysics. An interesting communication issue arises: to make objects from the remote universe meet the general public's imagery. The protagonists are not missing. Apart from black holes, there are new "monsters" from the sly: dark matter, dark energy, gamma ray flashes and active galactic cores are only waiting to be told. We pointed out that among the people there are those reacting either with trepidation or with fear to the invitation to those frontiers. Hence, one thing is not missing: the emotional sources to urge popular perception to search for what is "beyond". They could be promising if properly managed. It should be done with the necessary sensitiveness towards the attitudes of the public and the cultural dimensions of the new astrophysics, promoting a careful scientific criticism action. In order to make communication contribute successfully to the cultural placement $^{12}$ of astronomy, particular attention should be paid to the language used to popularise it and to the metaphors exploited, to avoid feeding unconsciously implicit circuits of meaning directly linked to the imagery ("Where would you travel into the universe?" "You take a space shuttle and you see what is there. I don't know the universe, I have never been to!" (Caterina, 16, classical secondary school student).

Someone who was there said: "I've seen things you people wouldn't even believe..." ${ }^{13}$ Well, now it is time to try.

Translated by Massimo Caregnato

\section{Notes and references}

${ }^{1}$ J.H. Falk, L.D. Dierking, The museum experience, Whalesback, Washington (1992).

2 C. Marsh, Visitors as learners: the role of emotions, ASTC online publication - Learning Research Task Force, http://www.astc.org.

3 G.E. Hein, Learning in the Museum, Routledge (1998).

4 Y. Castelfranchi, Per una paleontologia dell'immaginario scientifico, Jekyll.comm, 6 September 2003.

5 S. Macdonald, Cultural imaging among museum visitors: a case study, Museum Management and Curatorship 11 n.4 (1992) 401.

6 www.planetarioditorino.it.

E. Roletto, Apprendimento delle scienze didattiche e disciplinari, IriDiS 2002.

8 J. Diamond, Practical evaluation guide tools for museums and other informal educational settings, AltaMira Press, Walnut Creek (CA) (1999).

9 www.inaf.it/comunicati_stampa/cs160104/comunicato.htm.

10 M. Dussault, How do visitors understand the universe? Studies yield information on planning exhibitions and programs, Association of Science-Technology Centers Newsletter, May/June 1999. 
${ }^{11}$ P. Rodari, S. Cerrato, Progetto Preliminare ApritiCielo!, November 2003.

${ }^{12}$ J.M. Levy-Leblond, La pierre de touché. La science à l'éprouve..., Editions Gallimard, Paris 1996.

${ }^{13}$ Blade Runner di Ridley Scott, U.S.A. 1982, 117'. Starring Harrison Ford. The director's cut.

\section{Author}

Stefano Giovanardi, graduated in astronomy, has carried out research activities at the Space Telescope Science Institute in Baltimora and the Columbia University in New York. He has a Master's degree in science communication obtained at SISSA and is the scientific coordinator of the new Rome planetarium. E-mail: s.giovanardi@planetariodiroma.org. 